Revue internationale P.M.E.

Économie et gestion de la petite et moyenne entreprise

Revue

internationale

PME

\title{
La cognition comme objet d'étude dans la littérature scientifique sur la PME et l'entrepreneuriat
}

\section{Pierre Cossette}

Volume 13, numéro 1, 2000

URI : https://id.erudit.org/iderudit/1008668ar

DOI : https://doi.org/10.7202/1008668ar

Aller au sommaire du numéro

Éditeur(s)

Presses de l’Université du Québec

ISSN

0776-5436 (imprimé)

1918-9699 (numérique)

Découvrir la revue

Citer cet article

Cossette, P. (2000). La cognition comme objet d'étude dans la littérature scientifique sur la PME et l'entrepreneuriat. Revue internationale P.M.E., 13(1), 11-37. https://doi.org/10.7202/1008668ar
Résumé de l'article

Cette étude vise à mettre en évidence les caractéristiques des recherches portant sur des objets cognitifs dans la littérature scientifique sur la PME et l'entrepreneuriat. Fondée sur l'analyse de notices bibliographiques tirées de la banque de données ABI/INFORM de janvier 1987 à août 1994 et sur des articles auxquels renvoient ces notices, puis des articles publiés entre septembre 1994 et décembre 1998 dans les trois revues d'où provenaient la majorité des articles retenus dans l'étape précédente et, finalement, des articles parus dans la seule revue savante francophone spécialisée sur la PME, l'étude se construit à partir d'un cadre conceptuel constitué de quatre catégories d'objets cognitifs: les processus, les produits, les prédispositions et les procédés. L’analyse ayant trait aux revues concernées, aux objets cognitifs particuliers, aux échantillons, aux techniques de collecte et d'analyse des données ainsi qu'aux disciplines a porté sur 156 articles parus dans 28 revues scientifiques, sur 124 articles parus dans ETP, JBV et JSBM ainsi que sur 48 articles tirés de RI PME. 


\title{
La cognition comme objet d'étude dans la littérature scientifique sur la PME et l'entrepreneuriat ${ }^{\star}$
}

\author{
Pierre COSSETTE \\ Université du Québec à Montréal
}

MOTS CLÉS

Cognition, PME, Entrepreneuriat, Abi/Inform

\begin{abstract}
RÉSUMÉ
Cette étude vise à mettre en évidence les caractéristiques des recherches portant sur des objets cognitifs dans la littérature scientifique sur la PME et l'entrepreneuriat. Fondée sur l'analyse de notices bibliographiques tirées de la banque de données ABI/INFORM de janvier 1987 à août 1994 et sur des articles auxquels renvoient ces notices, puis des articles publiés entre septembre 1994 et décembre 1998 dans les trois revues d'où provenaient la majorité des articles retenus dans l'étape précédente et, finalement, des articles parus dans la seule revue savante francophone spécialisée sur la PME, l'étude se construit à partir d'un cadre conceptuel constitué de quatre catégories d'objets cognitifs: les processus, les produits, les prédispositions et les procédés. L'analyse ayant trait aux revues concernées, aux objets cognitifs particuliers, aux échantillons, aux techniques de collecte et d'analyse des données ainsi qu'aux disciplines a porté sur 156 articles parus dans 28 revues scientifiques, sur 124 articles parus dans ETP, JBV et JSBM ainsi que sur 48 articles tirés de RIPME.
\end{abstract}

\section{L'AUTEUR}

Pierre Cossette est professeur titulaire à l'École des sciences de la gestion de l'Université du Québec à Montréal. Après avoir complété un baccalauréat et une maîtrise ès arts en psychologie à l'UQTR, il a obtenu un M.B.A. et un Ph.D. en administration de l'Université Laval. La cognition et l'épistémologie sont au cœur de ses intérêts de recherche. Adresse: ESG-UQAM, Case postale 6192, Succ. Centre-Ville, Montréal (Québec) H3C 4R2. Courriel : cossette.pierre@uqam.ca

* Je tiens à remercier de façon particulière les lecteurs anonymes pour leurs précieux commentaires, particulièrement le lecteur 3 . 


\begin{abstract}
The aim of this study was to highlight the characteristics of research on cognitive objects in the scientific literature on small business and entrepreneurship. The study was based on data identified in three separate phases, the first concerned with bibliographic notices from the ABI/INFORM database for the period January 1987 to August 1994, together with the papers to which the notices referred, the second with papers published between September 1994 and December 1998 in the three journals in which the majority of the phase-one papers had been published, and the third with papers published in the only specialized French-language academic journal on small business. The study used a conceptual framework composed of four categories of cognitive objects, namely processes, products, predispositions and props. The analysis covered the journals in which the papers appeared, the specific cognitive objects studied, the samples used, the data collection and analysis techniques employed and the disciplines involved. In all, 156 papers published in 28 different scientific journals, 124 papers published in ETP, JBV and JSBM, and 48 papers published in RIPME were examined.
\end{abstract}

\title{
RESUMEN
}

Este estudio busca poner en evidencia las características de las investigaciones sobre objetos cognitivos en la literatura científica de la PyME y el liderazgo empresarial. Fundado inicialmente en el análisis de referencias bibliográficas obtenidas en la base de datos ABI/INFORM entre enero de 1987 y agosto de 1994, los artículos referidos, los artículos publicados entre septiembre de 1994 y diciembre de 1998 en las tres revistas de donde provenían la mayor parte de los artículos retenidos en la etapa anterior y, finalmente, los artículos publicados en la única revista francófona especializada en la PyME, el estudio se construye a partir de un cuadro conceptual constituido por cuatro categorías de objetos cognitivos: los procesos, los productos, las predisposiciones y los procedimientos. El análisis correspondiente a las revistas, los objetos cognitivos particulares, las muestras, las técnicas de recolección y análisis de la información asi como las diciplinas, se llevó a cabo sobre 156 artículos publicados en 28 revistas científicas, 124 artículos publicados en ETP, JBV y JSBM y sobre 48 artículos extraidos de RIPME.

\section{ZUSAMMENFASSUNG}

Diese Studie untersucht die Forschungsergebnisse in der wissenschaftlichen Literatur über kleine und mittlere Unternehmen und Unternehmertum. Die Studie stützte sich auf verschiedene Quellen: Zum einen dienten die bibliographischen Notizen der Datenbank ABI/INFORM von Januar 1987 bis August 1994 als Basis. Zum anderen wurden Artikel analysiert, welche zwischen September 1994 und Dezember 1998 in drei bekannten Zeitschriften veröffentlicht wurden. Und als dritte Quelle wurden Artikel aus der einzigen bekannten Zeitschrift im französischsprechendem Raum, welche auf KMU spezialisiert ist, analysiert. Die Studie verwendet einen konzeptionellen Rahmen, der vier kognitive Kategorien einteilt : Die Prozesse, die Produkte, die Veranlagungen und die Verfahren. Die Analyse bezieht sich auf die betroffenen Fachzeitschriften, auf kognitive Aspekte im allgemeinen, auf Beispiele, auf Techniken der Datenerhebung und Datenanalyse sowie auf Auszüge von 156 veröffentliche Artikel in 28 wissenschaftlichen Zeitschriften, auf 124 im ETP, JBV und JSBM erschienen Artikel sowie au 48 Artikel des RIPME. 


\section{Introduction}

Au cours des dernières années, l'intérêt pour la recherche sur la PME et l'entrepreneuriat a connu un essor considérable. Non seulement les articles publiés à ce propos dans les revues traditionnelles en sciences de l'administration en témoignent éloquemment, mais en outre les revues consacrées exclusivement à la diffusion de telles recherches sont de plus en plus nombreuses et reconnues dans la communauté scientifique. On pense, notamment, à Journal of Business Venturing (JBV), Entrepreneurship Theory and Practice (ETP), Journal of Small Business Management (JSBM) et, en français, à Revue internationale PME (RIPME). Il en est de même pour les colloques et congrès scientifiques parmi lesquels il faut nommer l'International Congress for Small Business et le Congrès international francophone de la PME. Compte tenu des particularités de ce type d'entreprises - la PME n'est pas une « petite » grande entreprise - (Dandridge, 1979; Welsh et White, 1981), on reconnaît généralement la nécessité d'élaborer des théories qui lui sont spécifiques (d'Amboise et Muldowney, 1988).

Cette activité scientifique intense a conduit un certain nombre de chercheurs reconnus à proposer différentes idées et orientations susceptibles de contribuer à l'enrichissement des connaissances dans le domaine de la PME et de l'entrepreneuriat. Parmi ceux-ci, il faut nommer Churchill et Lewis (1986), Wortman (1986, 1987), Ireland et Van Auken (1987), Bygrave (1989, 1993), Stevenson et Harmeling (1990), Herron et al. (1991, 1992), Bygrave et Hofer (1991), Hofer et Bygrave (1992) et MacMillan et Katz (1992). Cependant, la plupart d'entre eux, à l'exception notable de Bull et Willard (1993), n'attribuent pas au concept de cognition une importance particulière dans le renouvellement des connaissances sur la PME et l'entrepreneuriat. Pourtant, la dimension cognitive est considérée par un nombre grandissant de membres de la communauté scientifique comme essentielle à l'explication ou à la compréhension du fonctionnement des organisations. Elle a notamment été au cœur d'un certain nombre d'ouvrages largement diffusés dans les milieux de la recherche dont Mapping Strategic Thought (Huff, 1990), The Thinking Organization (Sims et Gioia, 1986) et The Social Psychology of Organizing (Weick, 1979). La cognition en sciences de l'administration a également fait l'objet de numéros spéciaux dans des revues scientifiques de premier ordre ; il faut mentionner, entre autres, le numéro spécial d'août 1994 de la revue Organization Science ainsi que ceux de mai 1992 et juillet 1989 de la revue Journal of Management Studies. Dans l'ensemble des sciences administratives, on accorde une attention soutenue et apparemment croissante à l'aspect cognitif. Audet et Déry (1996) prétendent même que c'est l'étude de la dimension cognitive qui caractérise la quatrième et plus récente «couche sédimentaire » du développement de l'épistémologie des sciences de l'administration, celle dite de la « dérivation conceptuelle », après celles de l'appropriation du discours scientiste, de la scientifisation des pratiques et de la révélation de la diversité des pratiques. Et rien n'indique que la 
prise en compte de cette dimension cognitive devrait se limiter à la seule étude des grandes organisations.

Dans le domaine de la PME et de l'entrepreneuriat, la place occupée par la cognition dans la production de connaissances est beaucoup moins évidente. En fait, nous connaissons très peu de chose sur l'importance et sur les caractéristiques des travaux à l'intérieur desquels la dimension cognitive est prise en considération. Cette étude veut remédier à cette situation. De façon plus spécifique, elle vise à mettre en évidence les caractéristiques des recherches portant sur des objets cognitifs dans la littérature scientifique sur la PME et l'entrepreneuriat. En soulevant des questions et des enjeux associés au développement des connaissances dans ce domaine particulier, les résultats de cette étude devraient stimuler la réflexion et la discussion dans notre communauté scientifique.

Dans cet article, j'apporterai d'abord quelques précisions sur la notion de cognition, avant de faire état des composantes du cadre conceptuel utilisé ici pour classer les travaux qui seront analysés. Nous présenterons ensuite les principaux éléments du cadre opératoire de cette étude en montrant qu'elle est fondée sur l'analyse de documents tirés de la banque de données ABI / INFORM, en l'occurrence des notices bibliographiques, et, subséquemment, les articles auxquels ces notices renvoient. Les résultats seront ensuite présentés et les revues qui auront publié le plus grand nombre d'articles ayant un objet cognitif seront examinées systématiquement en ce qui regarde leur comportement entre septembre 1994 et décembre 1998; il en sera de même pour les articles parus dans la seule revue savante francophone spécialisée en PME. Finalement, les résultats seront discutés dans la dernière partie de ce travail.

\section{Notion de cognition}

Voilà un concept difficile à manipuler. Notons d'entrée de jeu qu'il ne semble pas exister de nomenclature de termes cognitifs ni de consensus sur ce qui est « cognitif » et sur ce qui ne l'est pas (Meindl, Stubbart et Porac, 1994). Un bref regard sur les principaux termes associés à la cognition par différents auteurs dans un ouvrage dont la préparation fut dirigée par Andler (1992) à la suite d'un important colloque tenu au Centre culturel international de Cerisy-la-Salle illustre clairement l'ambiguïté de cette notion : langage, raisonnement, perception, planification, traitement de l'information, assimilation, stockage et accommodation de nouvelles informations, action finalisée, organisation conceptuelle, apprentissage, communication, aptitudes, propensions ou capacités cognitives du cerveau humain, entités mentales telles que processus et états mentaux (intentions, croyances, désirs, etc.), représentations mentales (par exemple, croyances, intentions, préférences) et représentations publiques (par exemple, signaux, énoncés, discours, textes). Les objets de nature cognitive semblent nombreux, diversifiés et non classifiés, bien que l'étude de la cognition renvoie toujours à l'étude de la pensée. 
Pour saisir différentes dimensions de cette notion en contexte organisationnel, Meindl et al. (1994) étaient d'avis que les études sur les organisations au sein desquelles la cognition occupait une place importante pouvaient se regrouper en deux grandes catégories, selon qu'elles portaient sur les processus ou les produits de la pensée. Ils reconnaissaient que peu d'études jusqu'à maintenant s'étaient intéressées simultanément à l'un et à l'autre. Par ailleurs, ils faisaient également mention de travaux menés sur des "procédés cognitifs » (cognitive aids) utilisés ou mis en place pour façonner ou représenter la pensée d'acteurs de l'organisation, tels que les systèmes d'aide à la décision et les systèmes d'information. Dans une autre étude, Schneider et Angelmar (1993) proposaient un cadre de référence constitué des trois éléments suivants pour faire état de la recherche sur la cognition dans les organisations : les structures cognitives (schèmes, croyances, scénarios, etc.), qui sont des modèles de prise de connaissance ou structures épistémologiques ; les processus cognitifs, qui renvoient à la manière particulière dont la connaissance est sélectionnée, organisée, transformée, emmagasinée et utilisée ; et, finalement, les styles cognitifs, qui mettent en évidence des différences individuelles, collectives ou organisationnelles dans la façon de traiter l'information. En somme, de tels modèles de classification autorisent à penser que l'étude de la cognition peut porter sur des processus, des produits, des prédispositions ou des procédés. Ce cadre conceptuel sera discuté de façon plus détaillée dans la prochaine section.

\section{Processus, produits, prédispositions et procédés cognitifs}

Les recherches centrées sur la dimension cognitive dans les organisations peuvent se situer dans au moins l'une des quatre catégories suivantes, selon leur objet d'étude : elles examinent soit les processus cognitifs, les produits cognitifs, les prédispositions cognitives ou les procédés cognitifs. Les deux premières catégories existaient dans les deux modèles présentés plus haut. Cependant, quelques précisions doivent être apportées au concept de «processus»; de plus, dans la présente recherche, les «produits » cognitifs ne seront pas limités aux « structures » cognitives. La troisième catégorie est très proche de ce que Schneider et Angelmar (1993) appellent les «styles » cognitifs, même si elle est plus large. Enfin, la catégorie des procédés cognitifs et celle des cognitive aids proposée par Meindl et al. paraissent équivalentes.

Les recherches sur les processus cognitifs ont pour objet les opérations, mécanismes, tâches ou activités mettant en évidence le fonctionnement de l'esprit et ayant trait à l'acquisition, au traitement, à la conservation, à la récupération, à la transformation ou à l'utilisation de l'information ou de la connaissance; elles visent essentiellement à mettre en relief comment les objets de la pensée (individuelle, collective, organisationnelle ou même sectorielle) se forment, se maintiennent ou se modifient. Il importe d'être très clair ici : l'étude d'un processus est l'étude du 
passage d'un état (ou "produit») cognitif à un autre; l'attention se trouve alors dirigée vers ce qui se passe entre un état cognitif au temps 1 et un état cognitif (de même nature ou non) au temps 2 . Un processus cognitif possède donc un caractère essentiellement dynamique ou évolutif. Considérons les deux exemples suivants, adaptés au contexte organisationnel. Un chercheur intéressé par les processus cognitifs pourrait se demander comment des croyances en arrivent à se transformer chez un ou plusieurs individus dans une organisation. Contrairement à celui qui est intéressé par les produits cognitifs, il ne se limitera pas à l'étude des croyances telles qu'elles existent à un moment donné, ni même à simplement comparer des croyances à deux moments différents : il mettra plutôt l'accent sur les caractéristiques du cheminement de la pensée (différentes étapes, biais cognitifs, apprentissage, etc.) suivi par les individus entre ces deux moments. Par ailleurs, un chercheur pourrait vouloir apporter des éclaicissements sur la démarche cognitive d'un individu le conduisant d'un état cognitif donné à un autre qui serait d'une nature fort différente, comme lors d'une prise de décision ${ }^{1}$. Plus précisément, il pourrait alors se demander comment cet individu passe d'une série d'options envisagées (état cognitif au temps 1) au choix d'une solution (état cognitif au temps 2). Il pourrait aussi souhaiter se concentrer sur un seul processus comme celui de la formulation de problème, l'analyse de l'environnement ou l'établissement de critères décisionnels ; son travail consisterait alors non pas à livrer le produit ou résultat de ces processus cognitifs (c'est-à-dire un problème formulé, un environnement analysé ou un ensemble de critères établis), mais à mettre en évidence leur déroulement particulier (c'est-à-dire le passage d'un problème non formulé à un problème formulé, etc.).

Dans les recherches axées sur les produits cognitifs, le chercheur s'intéresse au contenu de l'esprit plutôt qu'à son fonctionnement; il veut faire ressortir les caractéristiques de la pensée considérée comme le résultat d'un processus qui l'a précédée, un résultat qui existerait à un moment précis et qui serait plus ou moins stable. Les produits cognitifs peuvent être des structures mentales, comme des théories implicites, des scénarios ou des schèmes, c'est-à-dire des systèmes référentiels qui guident les individus dans l'interprétation qu'ils font des événements, dans leurs interventions et dans leurs prévisions. Ils n'ont toutefois pas à être des modèles plus ou moins généraux de prise de connaissance : ils peuvent renvoyer à des entités cognitives comme des significations, des perceptions, des interprétations,

1. Comme l'ont noté Schneider et Angelmar (1993), même en présumant que des processus cognitifs s'activent lors de l'accomplissement de tâches organisationnelles ou processus administratifs, comme la prise de décision ou l'évaluation du rendement, les deux ne doivent pas être confondus. Le chercheur s'intéressant à la prise de décision ne dirige pas nécessairement son attention vers les processus cognitifs qui interviennent au cours de ce processus administratif. Par exemple, il peut mettre l'accent sur la participation à la prise de décision (qui ? dans quelles circonstances ?...). 
des raisons, des anticipations, des motifs ${ }^{2}$ ou des intentions se rapportant à une situation particulière. Parmi les produits cognitifs dont l'étude pourra sembler pertinente en sciences de l'administration se trouvent les raisons données par des dirigeants pour expliquer leurs succès et leurs échecs, leurs perceptions des forces et faiblesses de leur organisation, les scénarios et théories implicites qui les guident dans diverses situations comme l'évaluation du rendement ainsi que tous les produits cognitifs associés au processus décisionnel (problème construit, sources d'information considérées plutôt que présumément « réelles », environnement analysé ou interprété, options envisagées, préférences mises de l'avant lors de l'évaluation des conséquences attribuées à ces options, etc.).

Certaines recherches portent sur les prédispositions cognitives des individus ; il s'agit là de caractéristiques personnelles pour lesquelles les psychologues manifestent souvent un grand intérêt. Sont contenues évidemment dans cette catégorie les études traitant des styles cognitifs, mais également les études sur les valeurs ou attitudes fondamentales préexistantes à la situation investiguée ainsi que sur toute autre disposition générale étroitement liée à la personnalité ou aux différences individuelles (niveau de tolérance à l'ambiguïté, niveau de complexité cognitive, etc.). Conformément aux propos de Schneider et Angelmar (1993) sur les styles cognitifs, on doit considérer que les collectivités et les organisations possèdent aussi des «prédispositions cognitives ». Il faut noter que certains de ces facteurs peuvent être des prédispositions cognitives ou des produits cognitifs, suivant la façon dont les auteurs en traitent. Par exemple, dans une recherche, si les attitudes représentent des tendances générales caractérisant la personnalité et mesurées à l'aide d'un test uniformisé, alors elles seront considérées comme des prédispositions cognitives. Par contre, si le terme «attitude » est plutôt utilisé pour désigner un état d'esprit construit et directement associé à une situation particulière se manifestant dans un contexte spatiotemporel donné (par exemple, une attitude plus ou moins favorable à l'exportation), alors l'attitude sera considérée comme un produit cognitif, un peu comme le serait une perception ou une intention. Le même raisonnement s' applique aux «valeurs»: elles peuvent être des convictions fondamentales ou générales guidant la vie d'un individu ou d'une collectivité, et dont l'origine remonte souvent à la tendre enfance et à l'éducation reçue ; elles peuvent également être des convictions plus ou moins partagées, façonnées dans l'entreprise et reflétant la culture d'une organisation donnée.

Finalement, il y a les procédés cognitifs. Les chercheurs qui se consacrent à l'étude des procédés cognitifs concentrent leurs efforts sur les moyens, outils, façons de faire ou procédures susceptibles d'aider à façonner ou représenter des produits

2. Les motifs sont des « raisons » généralement associées à la poursuite ou à l'évitement d'un but. Contrairement aux motivations ou besoins (qui ne sont pas considérées ici comme des entités cognitives), ils ne renvoient pas à des caractéristiques de la personnalité. 
ou des processus cognitifs ; ils démontrent comment ces procédés aident à orienter ou décrire la pensée. De telles études ont donc un caractère fondamentalement instrumental ou méthodologique. Sont généralement comprises dans cette catégorie les recherches sur les systèmes d'aide à la décision, notamment en ce qui a trait à la formulation des problèmes ; les études au sein desquelles la cartographie cognitive joue un rôle de premier plan, que ce soit comme outil d'aide à la communication, avec soi-même ou avec d'autres, ou comme outil d'analyse ; les travaux portant sur l'intelligence artificielle et, en particulier, sur l'ingénierie de la connaissance, dont l'un des résultats les plus spectaculaires est la mise au point de systèmes experts. Ne seront pas retenues dans la présente recherche les études portant, par exemple, sur l'utilisation plus ou moins grande d'équipements informatiques dans les PME ou, encore, sur l'impact de leur utilisation sur le rendement des entreprises.

C'est à partir de ces quatre grandes catégories que s'articule l'objet de la présente recherche : déterminer les recherches ayant pour objet, au moins en partie, l'étude de processus, produits, prédispositions ou procédés cognitifs dans la littérature scientifique sur la PME et l'entrepreneuriat, et relever les caractéristiques des travaux répertoriés. Dans la section suivante, les principaux éléments du cadre opératoire élaboré en fonction de l'objet de cette recherche seront décrits.

\section{Cadre opératoire}

Le contenu du cadre opératoire concerne les aspects méthodologiques de la recherche. Dans le cas présent, il faut d'abord mentionner que cette recherche s'est déroulée en deux temps. Dans un premier temps, elle a porté sur les articles répertoriés dans la banque de données ABI / INFORM entre janvier 1987 et août 1994³. À partir des résultats obtenus, j'ai poursuivi dans la même veine pour la période commençant en septembre 1994 et se terminant en décembre 1998, mais en me concentrant cette fois-ci uniquement sur les revues qui avaient publié le plus grand nombre de travaux ayant un objet cognitif, soit JBV, ETP et JSBM. De plus, tous les articles parus dans la seule revue savante francophone consacrée exclusivement à la PME, soit RIPME, ont été examinés depuis sa fondation en 1988 jusqu'à la fin de 1998. Mais avant de présenter concrètement la procédure suivie, notamment en ce qui a trait aux modalités de repérage et de sélection des notices bibliographiques tirées de la banque de données ABI / INFORM, il est nécessaire de fournir quelques éléments d'information sur cette banque de données à partir de laquelle la première phase de la recherche a été réalisée.

3. Les résultats de cette première phase ont été présentés lors du $\mathrm{II}^{\mathrm{e}}$ Congrès international francophone de la PME tenu à Paris en 1995 (voir Cossette, 1995), même s'ils n'ont pas fait l'objet d'une publication dans une revue scientifique. 
Selon le ABI/INFORM Ondisc User's Guide (1990), ABI / INFORM est une banque de données contenant des informations relatives aux articles publiés dans plus de 800 périodiques anglophones dont $75 \%$ proviennent des États-Unis. Ces périodiques, qui comprennent des revues professionnelles et scientifiques, couvrent les principaux domaines associés à la gestion et aux affaires, y compris la comptabilité, la finance, l'économique, la stratégie, le marketing, les ressources humaines, les systèmes d'information et le comportement organisationnel. ABI/INFORM fournit des notices bibliographiques constituées du titre de l'article et de son ou ses auteurs, de sa référence complète (nom de la revue dans laquelle il est publié, année, etc.), de mots clés, de " codes », représentant les domaines particuliers dans lesquels on peut le situer, et, finalement, d'un résumé de cet article.

La recherche informatisée sur disques compacts ou CD-ROM contenant les données d'ABI / INFORM a porté sur les articles publiés entre janvier 1987 et août 1994. Dans un premier temps, il fallait déterminer les termes précis à partir desquels le travail de repérage pourrait s'effectuer. D'un côté, en ce qui a trait à la PME et à l'entrepreneuriat, les termes small business et entrepreneurship furent retenus; ces deux concepts ne doivent pas être confondus et ont donné lieu à des recherches n'ayant parfois aucun lien entre elles (Wortman, 1986). Dans le cas présent, les recherches portant sur l'entrepreneuriat corporatif, ou « intrapreneuriat », n'ont pas été prises en compte, sauf si elles faisaient référence à l'intrapreneuriat dans la PME (voir, notamment Carrier, 1996). De l'autre côté, conformément à ce qui a déjà été dit sur la notion de cognition, les termes suivants furent considérés comme représentatifs du vocabulaire susceptible d'être employé par les chercheurs intéressés par les processus, les produits, les prédispositions ou les procédés cognitifs dans l'étude des organisations : cognition, representation, interpretation, scheme, construction, symbol, vision, perception, attribution, belief, intuition, thinking, meaning, making sense, receptivity, intention, objective, motive, frame, reason, sense making, ideology, decision, problem solving, expectation, preference, anticipation, attitude, value, opinion, information, knowledge, image, interest, goal, script, language, attention, memory, categorization et artificial intelligence. L'utilisation fréquente de la troncation (par exemple, cognit ?) permettait de repérer les mots ayant la même racine (cognitive, cognition, etc.). De toute évidence, cette liste ne représente pas un répertoire exhaustif de tous les termes cognitifs susceptibles d'être employés dans les recherches sur les organisations ; d'ailleurs, comme je l'ai mentionné précédemment, une telle nomenclature n'existe pas. Toutefois, il parait très improbable qu'un article portant sur l'organisation et ayant un objet cognitif ne contienne pas au moins un des termes de cette liste dans son résumé ou dans une autre des composantes de sa notice bibliographique, peu importe le paradigme, la perspective ou l'approche privilégiés dans cet article.

L'énoncé de recherche composé à partir de tous ces termes prit donc la forme suivante : (small business or entrepreneur?) and (cognit ? or represent ? or... or artificial intelligence). Cet énoncé de recherche visait à repérer toutes les notices 
bibliographiques d'ABI / INFORM (janvier 1987 à août 1994) contenant au moins un des termes de chacun des deux ensembles, que ce soit dans le titre, les mots clés, le résumé ou ailleurs. Compte tenu des limites imposées par les équipements informatiques, cet énoncé dut être scindé en plusieurs parties; plus de 30000 notices ont ainsi été visionnées sur écran. Avec une telle procédure, il était évident que de nombreuses notices devraient être écartées simplement parce que le terme accompagné de la troncation ferait apparaître des notices n'ayant rien à voir avec la dimension cognitive. Par exemple, avec le terme perce?, de nombreuses notices ont été repérées non parce qu'elles mettaient en évidence des perceptions, mais parce qu'elles contenaient le mot percentage ou percent.

Dans un deuxième temps, il fallait établir si la notice portait sur la réalisation d'une recherche publiée dans une revue s'adressant prioritairement à la communauté scientifique (par exemple, JBV), ou sur l'expression d'opinions, de réflexions, de convictions, d'analyses ou d'autres idées personnelles publiées dans une revue visant d'abord et avant tout une clientèle de praticiens (par exemple, Small Business Reports). Pour les besoins de cette recherche axée sur le développement ou renouvellement des connaissances, seules les notices de la première catégorie devaient être retenues; cependant, les notices provenant de revues visant explicitement les deux marchés (par exemple, Management International Review) étaient aussi conservées. La décision d'attribuer une notice à une catégorie ou à l'autre était prise à partir des indications données à ce propos dans le Cabell's Directory of Publishing Opportunities in Business and Economics ou encore dans la revue elle-même.

Enfin, la dernière tâche consistait à s' assurer que la recherche avait pour objet unique ou partiel l'étude d'au moins un processus, un produit, une prédisposition ou un procédé d'ordre cognitif; la simple allusion à de tels objets de recherche n'était pas suffisante. De même, une notice dans laquelle un ou plusieurs termes cognitifs étaient utilisés uniquement pour interpréter ou commenter les résultats d'une recherche portant sur un objet autre que cognitif était rejetée. En somme, en plus de vouloir apporter une contribution scientifique, la recherche devait focaliser, au moins en partie, sur un des objets cognitifs mentionnés. Ainsi, dès qu' un de ces objets cognitifs était traité comme une variable indépendante ou comme une variable dépendante, la notice était conservée, même si des objets non cognitifs étaient aussi investigués dans la recherche en question.

Cette décision de retenir ou non une notice était donc prise à partir d'une analyse du contenu de la notice. Le titre de l'article constituait fréquemment une excellente source de renseignements à cet effet. La présence d'au moins un terme cognitif dans la liste des mots clés, de plusieurs termes cognitifs dans le résumé ou encore d'un même terme cognitif revenant à plusieurs occasions laissaient également croire que la dimension cognitive occupait une place importante dans cette recherche. Par la suite, afin de prendre une décision encore plus éclairée, tous les articles 
auxquels renvoyaient les notices furent consultés sauf 13 , la revue contenant chacun d'eux n'ayant pu être trouvée malgré de nombreux efforts. C'était là une tâche fastidieuse, certes, mais qui permettait d'avoir accès à des informations importantes et parfois essentielles pour la présentation et l'analyse des articles en question. Près de la moitié des notices, donc des articles, ont ainsi été rejetées.

À partir des articles retenus, un tableau fut constitué. Ce tableau, dont on comprendra facilement qu'il ne peut être présenté ici faute d'espace ${ }^{4}$, contient des informations sur les références, les objets cognitifs étudiés, les caractéristiques des échantillons, les techniques de collecte et d'analyse des données utilisées et les disciplines concernées. Par la suite, les revues qui avaient publié le plus grand nombre d'articles ayant un objet cognitif entre 1987 et août 1994 ont été l'objet d'une attention spéciale relativement à leur contenu entre septembre 1994 et décembre 1998. Finalement, tous les articles publiés dans RIPME depuis sa fondation furent examinés de près, sur les mêmes dimensions que celles considérées au cours de la phase I. La section suivante sera consacrée à la présentation des résultats obtenus.

\section{Présentation et analyse des résultats}

De janvier 1987 à août 1994, 156 articles scientifiques ont porté au moins partiellement sur l'étude d'un processus, d'un produit, d'une prédisposition ou d'un procédé d'ordre cognitif, et cela, dans le cadre d'une recherche sur la PME ou l'entrepreneuriat (voir annexe). Certains trouveront peut-être que cet intérêt pour les objets cognitifs est plus grand qu'ils ne l'auraient cru et se demanderont pourquoi la cognition n'a pas été considérée plus clairement comme un facteur clé dans le développement de la théorie en entrepreneuriat et PME. Nous reviendrons plus loin sur cet aspect. La suite de cette partie sera consacrée à l'analyse de ces 156 articles à partir des six rubriques évoquées dans le cadre opératoire, à l'analyse des articles de même nature parus dans ETP, JBV et JSBM entre septembre 1994 et décembre 1998 ainsi qu'à l'analyse des articles ayant un objet cognitif publiés dans RIPME depuis sa fondation en 1988 jusqu'en décembre 1998.

\section{Références}

Le tableau 1 montre le nombre d'articles publiés dans chaque revue de 1987 à août 1994. On voit d'abord que la majorité des articles (119/156, soit $76 \%)$ proviennent des trois seules revues du tableau qui soient spécialisées sur la PME et l'entrepreneuriat soit, dans l'ordre, JSBM, JBV et ETP. On constate aussi le nombre important de revues (28) dans lesquelles sont publiés ces articles. Également, le nombre de ces

4. Ce tableau est présenté intégralement dans Cossette (1995, p. 508-524). 
articles est remarquablement stable d'une année à l'autre, si l'on exclut 1987 et 1994 (dans ce dernier cas, il devrait y avoir une diminution importante si la tendance se maintient dans les quatre derniers mois de 1994).

\section{TABLEAU 1}

Nombre d'articles publiés par revue de 1987 à août 1994

\begin{tabular}{llllllllll}
\hline REVUES & 1987 & 1988 & 1989 & 1990 & 1991 & 1992 & 1993 & $\begin{array}{c}1994 \\
\text { janv.-août }\end{array}$ \\
\hline
\end{tabular}

Academy of Management Executive

(AME)*

Academy of Management Review

(AMR)

Accounting and Business Research

(ABR)

Administrative Science Quarterly

(ASQ)

Akron Business and Economic Review

(ABER)

Decision Support Systems (DSS)

Entrepreneurship Theory and Practice

(ETP) (anciennement American

Journal of Small Business)

Industrial Marketing Management

(IMM)*

Information Age (IA)*

Journal of Applied Business Research

(JABR)

Journal of Banking and Finance

(JBF)*

Journal of Business Communication

(JBC)

Journal of Business Ethics

Journal of Business Finance

and Accounting (JBFA)

Journal of Business Venturing (JBV)

Journal of Economic Behavior

and Organization (JEBO)

Journal of Economic Psychology (JEP)

Journal of Management Studies (JMS)

Journal of Occupational Psychology

(JOP)

Journal of Small Business Management

(JSBM)

Journal of the Operational Research

Society (JORS)*

Journal of Travel Research (JTR)*

Management International Review

(MIR)*

Managerial Finance (MF)

MIS Quarterly (MISQ)*

Organizational Dynamics (OD)

Strategic Management Journal

Technovation $(\mathrm{T})^{*}$

$\begin{array}{llll}1 & & & 1 \\ & & & 2 \\ & 1 & 1 & 2 \\ 1 & & 1 \\ & 1 & 1 & 2 \\ & & 1 & 1\end{array}$

\begin{tabular}{|c|c|c|c|c|c|c|c|}
\hline \multirow{5}{*}{\multicolumn{2}{|c|}{4}} & 2 & 2 & 3 & 5 & 3 & 3 \\
\hline & & & 1 & 1 & & & \\
\hline & & & & 1 & 1 & & \\
\hline & & & & 1 & & & \\
\hline & & & 1 & & 1 & & \\
\hline
\end{tabular}

\begin{tabular}{llllllllll}
\hline Total & 10 & 21 & 20 & 24 & 24 & 23 & 24 & 10 & 156 \\
\hline
\end{tabular}

* Revue s'adressant à la fois aux universitaires et aux praticiens. 
Trois autres points méritent d'être signalés. D'abord, en se fondant sur la classification proposée dans l'étude de MacMillan (1993), 39 articles proviennent de revues de la catégorie 4 (la plus forte), aucun de la catégorie 3, 27 de la catégorie 2 et 62 de la catégorie 1 (la plus faible). Les autres articles ont été publiés dans des revues qui, dans la recherche de MacMillan, n’ont pas été considérées parmi les plus pertinentes pour le chercheur désirant publier dans le domaine de l'entrepreneuriat. Ces résultats ne permettent pas de conclure que les articles portant sur la cognition dans les PME paraissent surtout dans des revues reconnues comme particulièrement prestigieuses, ni l'inverse. Ensuite, en ce qui a trait aux articles « conceptuels », ils sont au nombre de 21 et sont parus dans les six revues suivantes : ETP (10 [7 depuis 1992], soit près de la moitié de tous les articles de la présente recherche publiés dans cette revue), JSBM (4, tous avant 1990), JEBO (3), AMR (2), JBV (1) et DSS (1). Au total, plus de la moitié de ces articles conceptuels sont parus depuis 1992 et aucun d'eux ne provient d'une revue s'adressant à la fois aux universitaires et aux praticiens. Le tiers de ces articles sont parus dans des revues qui ne sont pas spécialisées sur la PME et l'entrepreneuriat. Finalement, seulement 10 des 156 articles ont été publiés dans les neuf revues s'adressant à la fois aux universitaires et aux praticiens ; tous, sauf un, sont parus avant 1992. Les recherches scientifiques portant sur la PME et ayant un objet cognitif sont donc principalement publiées dans des revues visant uniquement un lectorat d'universitaires.

Si l'on s'attarde maintenant au comportement des trois revues principales à partir de septembre 1994 jusqu'à décembre 1998, on obtient le tableau 2. On constate d'abord que JSBM est encore en tête de peloton, suivi de JSV (qui publie six numéros par volume alors que les deux autres n'en publient que quatre) et ETP, même si les différences entre les trois revues se sont grandement atténuées. De plus, chacune de ces revues publie en moyenne, annuellement, davantage d'articles ayant un objet cognitif qu'au cours de la période précédente. Finalement, on note que les fluctuations ne sont pas très grandes d'une année à l'autre.

TABleau 2

Nombre d'articles publiés dans ETP, JBV et JSBM de septembre 1994 à 1998

\begin{tabular}{lccccrr}
\hline REVUES & Sept.-déc. & 1995 & 1996 & 1997 & 1998 & TOTAL \\
\hline ETP & 2 & 10 & 7 & 9 & 4 & 32 \\
JBV & 3 & 9 & 8 & 9 & 14 & 43 \\
JSBM & 6 & 13 & 13 & 12 & 5 & 49 \\
\hline TOTAL & $\mathbf{1 1}$ & $\mathbf{3 2}$ & $\mathbf{2 8}$ & $\mathbf{3 0}$ & $\mathbf{2 3}$ & $\mathbf{1 2 4}$ \\
\hline
\end{tabular}

En ce qui a trait à RIPME, comme le fait voir le tableau 3, l'intérêt pour les objets cognitifs se maintient au fil des ans. Le nombre d'articles qui s'y consacrent 
au moins partiellement depuis 1988 est plus faible que dans les trois revues anglophones précitées, mais il faut se rappeler que RIPME ne publie que trois numéros par année (deux en 1989), dont un numéro double, et que près de la moitié des textes ont un lien direct avec l'économique (Cossette, 1997), une discipline qui se prête moins aux objets cognitifs.

TABleau 3

Nombre d'articles publiés dans RIPME de 1988 à 1998

\begin{tabular}{cccccccccccc}
\hline 1988 & 1989 & 1990 & 1991 & 1992 & 1993 & 1994 & 1995 & 1996 & 1997 & 1998 & TOTAL \\
\hline 5 & 2 & 8 & 4 & 4 & 3 & 3 & 2 & 3 & 3 & 11 & 48 \\
\hline
\end{tabular}

\section{Objets cognitifs}

Comme le montre le tableau 4, ce sont les produits cognitifs qui, très nettement, reçoivent le plus d'attention des chercheurs entre 1987 et août 1994. Dans chaque catégorie, le nombre d'articles varie peu au fil des ans, si ce n'est qu'un peu plus d'intérêt semble être accordé depuis 1991 aux prédispositions cognitives.

TABLEAU 4

Nombre d'articles publiés par catégorie d'objet cognitif de 1987 à août 1994

\begin{tabular}{lrrrrrrrrrr}
\hline & 1987 & 1988 & 1989 & 1990 & 1991 & 1992 & 1993 & $\begin{array}{l}\text { 1994 } \\
\text { janv.- } \\
\text { août }\end{array}$ & TOTAL \\
\hline Processus & 1 & 3 & & 2 & 1 & 4 & & & 11 \\
Produit & 6 & 17 & 14 & 21 & 17 & 15 & 20 & 9 & 119 \\
Prédisposition & 1 & 1 & 2 & 2 & 4 & 3 & 3 & 1 & 17 \\
Procédé & 2 & & 4 & 1 & 2 & 1 & 1 & & 11 \\
\hline & 10 & 21 & 20 & $26^{*}$ & 24 & 23 & 24 & 10 & $158^{*}$ \\
\hline
\end{tabular}

* Ce chiffre diffère de celui qu'on trouve dans le tableau 1 étant donné que deux recherches en 1990 ont porté chacune sur deux objets cognitifs.

Les objets cognitifs particuliers étudiés dans chaque catégorie sont variés même si certains d'entre eux reviennent plus souvent que d'autres. Ainsi, les recherches axées sur les processus tendent principalement à décrire la démarche cognitive suivie au cours d'une prise de décision ou d'une résolution de problème. Celles sur les prédispositions visent surtout à déterminer les valeurs ou attitudes fondamentales d'entrepreneurs ou d'étudiants, ou encore leur style de prise de décision. Les recherches tournées vers l'étude de procédés sont souvent prescriptives et montrent comment un modèle ou une technique peut aider à penser, généralement dans un cadre décisionnel et dans des domaines très divers (GRH, FIN / CTB, MRK, 
SIO, etc.) Mais ce sont les produits cognitifs qui retiennent le plus l'attention : trois recherches sur quatre y sont consacrées. Les raisons ou motifs évoqués en rapport avec le démarrage d'une entreprise, les critères ou facteurs considérés comme importants dans une situation de prise de décision et, surtout, les perceptions (de l'importance des sources d'information, de l'environnement, des problèmes de l'entreprise, des freins à l'exportation, etc.) sont parmi les produits cognitifs les plus recherchés.

L'intérêt particulier accordé aux perceptions mérite qu'on s'y attarde un peu. Comme l'ont bien fait ressortir Smircich et Stubbart (1985), le terme perception suggère l'existence d'un environnement objectif, d'une réalité dure qu'on peut appréhender en faisant plus ou moins d'erreurs. Lié aux stimulations sensorielles, il laisse croire que le sujet ne fait que réagir à une réalité considérée comme externe et indépendante de lui-même. De tels présupposés n'existent pas lorsqu'on fait plutôt appel au concept de représentation dont on peut noter l'absence quasi totale de la recherche sur la PME et l'entrepreneuriat.

Depuis septembre 1994, en se limitant à ETP, JVB et JSBM, l'ordre d'importance des quatre objets cognitifs est resté le même, malgré l'intérêt grandissant pour les processus et les prédispositions (voir tableau 5); la stabilité se maintient au fil des ans et à l'intérieur de chaque catégorie. Trois autres constatations peuvent être faites : 12 des 16 recherches réalisées sur des processus cognitifs ont été publiées dans ETP (aucune dans JSBM) ; plus de recherches ont porté sur des prédispositions cognitives de septembre 1994 à décembre 1998 dans ces trois revues que dans la période allant de janvier 1987 à août 1994 pour toutes les revues répertoriées dans ABI/INFORM ; aucune recherche sur les procédés cognitifs n'est parue dans l'une ou l'autre de ces trois revues depuis 1996.

TABleau 5

Nombre d'articles publiés par catégorie d'objet cognitif dans ETP, JBV et JSBM de septembre 1994 à décembre 1998

\begin{tabular}{lcccccc}
\hline & 1994 & 1995 & 1996 & 1997 & 1998 & TOTAL \\
\hline Processus & 1 & 6 & 2 & 5 & 2 & 16 \\
Produit & 8 & 23 & 24 & 19 & 17 & 91 \\
Prédisposition & 1 & 5 & 5 & 7 & 7 & 25 \\
Procédé & 1 & 1 & & & & 2 \\
\hline & 11 & 35 & 31 & 31 & 26 & $134 *$ \\
\hline
\end{tabular}

* Ce chiffre diffère de celui qu'on trouve dans le tableau 2, étant donné que 10 recherches ont porté chacune sur deux objets cognitifs.

Les objets cognitifs particuliers ne paraissent pas avoir changé beaucoup. L'étude des produits cognitifs s'est encore fréquemment centrée sur les raisons données pour se lancer en affaires et sur la perception de l'environnement. Les 
valeurs personnelles et les attitudes profondes à l'égard de l'entrepreneuriat constituent des prédispositions qui ont aussi fait l'objet de plusieurs recherches.

Dans RIPME, le tableau 6 montre que l'étude des procédés cognitifs suscite davantage d'intérêt, se situant immédiatement après celle des produits cognitifs. Parmi les procédés qui ont fait l'objet de recherches se trouvent les méthodes d'aide à l'évaluation de projets d'entreprise ou d'accompagnement d'entrepreneurs et les outils d'aide à la réflexion stratégique. Une seule recherche a été consacrée à l'étude des processus cognitifs. Les produits cognitifs qui ont reçu le plus d'attention ne semblent pas vraiment différents de ceux dont nous avons fait état précédemment (motifs pour lancer une entreprise et perception de l'environnement.

TABleau 6

Nombre d'articles publiés par catégorie d'objet cognitif dans RIPME de 1988 à 1998

\begin{tabular}{|c|c|c|c|c|c|c|c|c|c|c|c|c|}
\hline & 1988 & 1989 & 1990 & 1991 & 1992 & 1993 & 1994 & 1995 & 1996 & 1997 & 1998 & TOTAL \\
\hline \multicolumn{13}{|l|}{ Processus } \\
\hline Produit & 5 & 2 & 7 & 2 & 2 & 2 & 3 & 1 & 2 & 1 & 10 & 37 \\
\hline Prédisposition & & & & 1 & & & & 1 & & 1 & 2 & 5 \\
\hline \multirow[t]{2}{*}{ Procédé } & & & 1 & 1 & 2 & 1 & & & 1 & 1 & & 7 \\
\hline & 5 & 2 & 8 & 4 & 4 & 3 & 3 & 2 & 3 & 2 & 13 & $50 *$ \\
\hline
\end{tabular}

* Ce chiffre diffère de celui apparaissant au tableau 4, car une recherche a porté sur trois objets cognitifs.

\section{Échantillons}

Dans le cas des recherches empiriques, la grandeur de l'échantillon varie d'une recherche à l'autre, mais il est rarement inférieur à 50 individus et souvent supérieur à 100 . Le niveau d'étude est presque toujours individuel; il semble que la cognition aux niveaux collectif, organisationnel et même industriel, cognition qu'on ne pourrait alors considérer comme le résultat d'une simple agrégation de pensées individuelles (Schneider et Angelmar, 1993), intéresse peu les chercheurs en PME et entrepreneuriat. Par ailleurs, la diversité des sujets constituant les échantillons est remarquable. Bien que les propriétaires-dirigeants soient les plus sollicités, beaucoup d'autres acceptent de se mettre au service de la production de connaissance : gestionnaires, employés, investisseurs, prêteurs, clients, comptables, spécialistes en marketing, professeurs, étudiants, etc., acceptent d'agir comme sujets dans des recherches portant sur la cognition dans le domaine de la PME et de l'entrepreneuriat. Il faut également signaler que ces sujets proviennent d'une grande variété d'entreprises ; la plupart des secteurs économiques sont représentés et, ce qui peut surprendre, les recherches répertoriées ont été réalisées dans une multitude de pays (au moins une quinzaine). 
En ce qui concerne plus précisément ETP, JBV et JSBM de septembre 1994 à décembre 1998, les recherches empiriques comptant moins de 30 sujets sont rares : six pour ETP, quatre pour JBV et une seule pour JSBM. La recherche dans les PME de haute technologie, en particulier dans le domaine de l'informatique, semble occuper une place grandissante; il en est de même pour les franchises. Les sujets sont également très nombreux dans les recherches publiées dans RIPME et proviennent d'une multitude de pays.

\section{Techniques de collecte de données}

Dans les articles répertoriés au cours de la première phase de cette recherche, c'est très clairement le questionnaire envoyé par la poste qui est l'outil le plus fréquemment utilisé. Pour ceux qui croient que la cognition possède un caractère fondamentalement idiosyncrasique (Cossette, 1994b), l'emploi de cette technique structurante, combiné au fait que les données sont recueillies dans un contexte impersonnel ${ }^{5}$, n'est pas sans inquiéter. En effet, dans ce questionnaire dominent les questions à évaluation (échelles de Likert) ou à éventail de réponses (plusieurs réponses suggérées parmi lesquelles le sujet fait un choix) qui fournissent au sujet un cadre de référence qui n'est pas le sien mais celui du chercheur, contrairement à ce qui se produit lorsqu'on fait appel à des techniques comme l'entrevue en profondeur, les phrases à compléter et la verbalisation des pensées (avec enregistrement) au cours d'une tâche.

C'est également avec des questions à évaluation que les objets cognitifs ont surtout été étudiés dans les recherches analysées au cours de la seconde phase de ce travail : dans presque tous les cas pour la revue JSBM, dans 31 des 43 recherches de JBV, mais moins fréquemment dans ETP où les approches plus qualitatives semblent mieux acceptées. Cette propension à utiliser les échelles de Likert s'observe aussi dans RIPME, même si les chercheurs font davantage appel aux questions à éventail de réponses et que les questionnaires y sont administrés plus souvent qu'ailleurs lors de rencontres individuelles avec les sujets.

\section{Techniques d'analyse des données}

Compte tenu de ce qui a été dit dans les sections sur les échantillons et les techniques de collecte des données, on ne se surprendra guère d'une utilisation massive des statistiques pour analyser les données obtenues. Dans les articles publiés entre janvier 1987 et août 1994, l'analyse de contenu n'a été employée qu'à quelques

5. On ne peut même pas être certain que c'est le sujet visé qui répond effectivement au questionnaire. L'absence d'un contact avec le chercheur n'est probablement pas étrangère au fait que le taux de réponse avec cette technique se situe généralement autour de $20 \%$... 
occasions. En ce qui a trait aux statistiques utilisées, il faut d'abord noter la présence de statistiques descriptives dans la majorité des recherches ; ces statistiques empruntent des méthodes graphiques (distribution de fréquences, histogramme, etc.) ou encore des méthodes de calcul numérique fournissant des indices de position ou de dispersion (moyenne, écart type, mode, médiane, rang, pourcentage, etc.). Certaines techniques multivariées ont aussi pour objectif de décrire. L'utilisation de ces techniques beaucoup plus complexes, comme l'analyse discriminante, l'analyse factorielle et l'analyse de regroupement, était en nette progression dans les recherches portant sur la cognition dans la PME : de janvier 1992 à août 1994, elles ont été utilisées à 23 occasions alors qu'elles l'avaient été seulement 21 fois dans les cinq années précédentes. L'analyse factorielle est la plus populaire de ces techniques : on s'en est servi dans 27 recherches, alors qu'on a utilisé l'analyse discriminante 9 fois et l'analyse de regroupement 8 fois.

L'utilisation de statistiques explicatives, c'est-à-dire celles associées à l'inférence statistique, a été en hausse jusqu'en 1991 ( 25 de ces statistiques avaient été employées cette année-là), pour être en baisse jusqu'en 1994 ; les différences ne sont toutefois pas assez importantes pour y voir l'effet d'un changement de paradigme. Par ailleurs, les techniques multivariées visant à expliquer (analyse de régression et analyse de variance) sont plus utilisées depuis 1991. Rappelons que cette tendance existait concernant l'utilisation des techniques multivariées descriptives ; le degré de sophistication des techniques d'analyse de données est donc de plus en plus élevé, conformément au souhait exprimé par Wortman (1986) mais contrairement à ce que laissait entendre Bygrave (1989). Plus récemment, Bygrave et Hofer (1991) proposaient de délaisser l'analyse de régression : « [...] l'analyse de régression est réductionniste, alors que l'entrepreneuriat est holistique [...] son utilisation repose sur l'idée que les modèles proposés sont stables et contiennent peu de variables » (p. 20).

Trois autres points doivent être mis en évidence relativement à l'utilisation des statistiques explicatives telle qu'elle s'est manifestée au cours de la première phase de cette recherche : les statistiques multivariées sont à peu près aussi utilisées que les statistiques bivariées ; les statistiques paramétriques bivariées sont près de deux fois plus utilisées que les statistiques non paramétriques bivariées ; les statistiques cherchant essentiellement à établir une relation entre des variables $(r$, rho, khi carré, analyse de régression, etc.) sont utilisées un peu plus fréquemment (en 78 occasions contre 66) que les statistiques visant à établir des relations entre des groupes en comparant des moyennes ( $t$, analyse de variance, etc.).

Dans ETP, JBV et JSBM entre septembre 1994 et décembre 1998, il n'y a pas de changement majeur dans la façon d'analyser les données des recherches portant sur des sujets cognitifs. Au cours de cette période, les techniques multivariées descriptives ont été employées en 37 occasions (dont 26 pour l'analyse factorielle), principalement dans JBV $(n=20)$, ensuite dans JSBM $(n=13)$ et, 
finalement, dans $\operatorname{ETP}(n=4)$. Quant aux statistiques multivariées explicatives, l'analyse de régression a été utilisée 42 fois ( 25 dans JBV, 10 dans JSBM et 7 dans ETP) et l' analyse de variance 27 fois ( 15 dans JSBM, 10 dans JBV et 2 dans ETP). Bref, la progression dans l'utilisation des techniques multivariées ne semble pas vouloir s'arrêter, loin de là. Par contre, cette progression n'existe pas dans RIPME: les techniques multivariées ont été employées plus souvent au cours des cinq premières années de son existence qu'au cours des six dernières. Au total, les chercheurs francophones s'intéressant aux objets cognitifs recourent très peu aux techniques multivariées : en 11 ans, ils ont employé 18 fois l'une ou l'autre des techniques descriptives et seulement 4 fois les techniques explicatives (trois fois l'analyse de régression et une fois l'analyse de variance).

\section{Disciplines}

De toute évidence, l'étude de la cognition dans la PME n'est pas la chasse gardée du sous-champ «comportement organisationnel », même si ce dernier domine largement. Il est intéressant de noter que le domaine de la finance et de la comptabilité a été rattaché à 26 articles tirés d'ABI / INFORM, soit le deuxième plus grand nombre après le domaine du comportement organisationnel. Les chiffres ne constituent pas une réalité indépendante des représentations qu'on leur associe... Soulignons également que la stratégie, le marketing et les systèmes d'information organisationnels sont des domaines auxquels sont reliés, partiellement ou en totalité, au moins 10 articles dans chacun des cas. Même s'il est parfois très difficile d'associer une recherche à une discipline particulière, les mêmes tendances semblent se dessiner dans ETP, JBV, JSBM et RIPME.

\section{Discussion et conclusion}

L'objectif de cette étude était de mettre en évidence les caractéristiques des recherches portant sur des objets cognitifs dans la littérature scientifique sur la PME et l'entrepreneuriat. Les résultats ont montré que c'est peut-être la diversité qui caractérise le mieux l'ensemble des recherches sur la cognition dans la PME. Les revues dans lesquelles les articles ont été publiés, les objets cognitifs, les échantillons, les techniques de collecte et d'analyse de données ainsi que les disciplines concernées sont en effet d'une grande variété. Parfois même, surtout en ce qui a trait aux objets cognitifs particuliers qui ont été étudiés, il se dégage une impression de fragmentation tellement il semble y avoir peu de rapports entre eux ${ }^{6}$. La même

6. Déry (1989) avait conclu que la fragmentation était aussi « le trait principal de la structuration objectale de la problématique de la décision dans la revue ASQ» (p. 390). Il pourrait s'agir là d'une caractéristique générale des recherches en sciences de l'administration. 
constatation avait d'ailleurs été faite par Churchill et Lewis (1986) il y a une dizaine d'années relativement à l'ensemble de la recherche en entrepreneuriat : «[...] les voies de recherche sont fragmentées, créatives et variées » (p. 334). Les résultats obtenus, bien sûr, indiquent certaines voies de recherche. Cependant, des commentaires doivent d'abord être apportés relativement aux «limites » de la présente étude.

La recherche documentaire au cours de la première phase a été effectuée uniquement à partir de la base de données $\mathrm{ABI} / \mathrm{INFORM}$. Bien qu'il soit reconnu que cette banque de données constitue un excellent répertoire des articles scientifiques parus en sciences de l'administration, il faut noter qu'elle ne contient pas, dans sa version courante, les titres de 100 revues internationales dont les articles ne sont répertoriés que dans la version «complète » (global) et plus coûteuse d'ABI/ INFORM. Ainsi, les articles provenant d'au moins une revue bien connue, même si elle est rangée par les experts dans la classe la plus faible des revues (classe 4, selon l'étude de MacMillan, 1993), soit l'International Small Business Journal, ne figurent pas dans la version standard. Finalement, la présente étude n'a pas tenu compte des recherches publiées dans des ouvrages ou actes rédigés à la suite de congrès scientifiques ; le contenu d'ABI/ INFORM ne provient que de périodiques.

On doit aussi rappeler que les termes présents dans les énoncés de recherche et associés à la dimension cognitive des organisations ont été déterminés de façon « intuitive » étant donné qu'il n'existe pas de modèle ou de typologie présentant une manière particulière d'organiser ou de structurer conceptuellement le domaine de la cognition. Bien que les termes utilisés dans la présente recherche semblent constituer un répertoire assez étendu des termes cognitifs employés en sciences de l'administration, un autre chercheur n'y souscrirait peut-être pas dans sa totalité. Ceci fait ressortir la nécessité d'élaborer des modèles ou typologies qui permettraient d'enrichir l'étude de la dimension cognitive dans les organisations, quelle que soit leur taille. Parallèlement, on peut penser que certaines recherches portent effectivement sur des objets cognitifs, en particulier sur des perceptions, même si le titre ou le résumé n'en faisaient pas état ; elles n'ont donc pu être repérées à partir de l'énoncé qui a guidé le travail de recherche sur ABI/INFORM. Cependant, tous les articles publiés dans les revues reconnues comme les plus importantes dans le domaine de la PME et de l'entrepreneuriat - ETP, JBV et JSBM - ont été regardés de très près pour voir si certains d'entre eux auraient dû être répertoriés dans cette étude même si le titre et le résumé ne contenaient pas les termes cognitifs utilisés ici : aucun des articles non inclus n'aurait dû être répertorié pour cette recherche.

Une voie de recherche que les résultats de cette étude suggèrent d'explorer concerne la comparaison de l'intérêt des scientifiques pour les objets cognitifs à celui que leur accordent les praticiens. Il est loin d'être évident que les préoccupations des chercheurs correspondent à celles des praticiens, notamment dans le domaine des PME (à ce propos, voir, entre autres, Ireland et Van Auken, 1987; Brockhaus, 1987; Sandberg et Gatewood, 1991). En comparant les articles des 
revues s'adressant principalement aux premiers à ceux des revues s'adressant principalement aux seconds, on pourrait se poser plusieurs questions dont les suivantes : Sont-ils plus nombreux dans un cas que dans l'autre ? Portent-ils sur les mêmes objets cognitifs (processus, produits, prédispositions, procédés) ? L'intérêt exprimé pour différents objets cognitifs dans les revues savantes précède-t-il ou suit-il celui affiché dans les revues professionnelles?

Mais le plus important est peut-être de reconnaitre que les travaux portant sur des objets cognitifs et effectués dans le cadre d'études sur la PME et l'entrepreneuriat ont presque toujours été réalisés à partir d'une perspective qu'on pourrait qualifier d' «objectiviste »; cette orientation y est probablement encore plus dominante que dans les recherches sur les grandes organisations. On semble présumer qu'on peut et doit étudier la réalité - y compris la réalité cognitive - en toute neutralité, « de l'extérieur, comme si on pouvait l'observer à travers une fenêtre » (Evered et Louis, 1981), afin de découvrir des régularités ou lois de manière à pouvoir prédire, objectif ultime de la science développée dans le sillage du positivisme. Au plan méthodologique, l'approche nomothétique est privilégiée. Idéalement, les sujets doivent être nombreux et constituer un échantillon statistiquement représentatif d'une population donnée et clairement précisée. On favorise l'utilisation de tests ou de questionnaires, uniformisés si possible, dont les résultats sont classifiés dans des catégories prédéterminées, et analysés à l'aide de techniques quantitatives. C'est là la voie pointée en 1987 par Sexton qui soulignait l'importance de rechercher des relations causales entre les événements, de s'assurer que les résultats peuvent être reproduits, de bien échantillonner et généraliser ${ }^{7}$ en conséquence, d'avoir des instruments de mesure valides et d'effectuer des analyses mathématiques sophistiquées. D'autres également proposent essentiellement d'emprunter la même direction. Ainsi, Low et MacMillan (1988) insistent pour faire de la causalité la préoccupation centrale des chercheurs en entrepreneuriat et déplorent l'approche adoptée jusqu'à maintenant : «Le peu de recherches réalisées à partir d'une approche expérimentale est une autre indication de la lenteur des progrès accomplis au plan théorique dans le domaine de l'entrepreneuriat » (p. 154).

Pourtant, en 1987, Vesper reconnaissait que le progrès réalisé dans la recherche sur l'entrepreneuriat était difficile à évaluer et que des critères tels que la valeur prédictive associée à la génération et au test d'hypothèses n'étaient peut-être pas appropriés en recherche sociale, y compris en entrepreneuriat; il notait que le progrès nécessitait souvent le rejet de paradigmes dominants. Les recherches des dernières années ne permettent pas de croire que ses propos aient eu l'impact souhaité.

7. Même lorsqu'on propose l'étude en profondeur de cas particuliers comme stratégie de recherche (voir, notamment, Romano, 1989), on insiste généralement sur l'importance de la généralisation des résultats. Le plus souvent, on considère que les méthodes qualitatives servent à générer des hypothèses qui seront éventuellement mises à l'épreuve. 
Dans la même veine, Hisrich (1988) affirmait : «Il serait surprenant [...] qu'une théorie scientifique prédictive de l'entrepreneuriat voie éventuellement le jour » (p. 3). Également, Bygrave (1989) déplorait que les études sur l'entrepreneuriat soient surtout réalisées à l'aide de méthodes et de théories empruntées à d'autres sciences, en particulier aux sciences dures, ce qu'il expliquait par l'état peu avancé des connaissances en entrepreneuriat (et aussi, en partie, par le fait que plusieurs de ses ténors ont une formation de base en génie ou en mathématiques). Les conclusions de Bygrave et Hofer (1991), Hofer et Bygrave (1992) ainsi que de Stevenson et Harmeling (1990) vont dans le même sens. Plus récemment, Busenitz et Lau (1996) proposaient l'adoption d'un modèle cognitif pour mieux comprendre l'entrepreneuriat, en se centrant sur la «façon de penser» de l'entrepreneur et en particulier sur ses «schèmes ». De même, Jelinek et Litterer (1995) insistaient sur la nécessité d'abandonner la perspective traditionnelle déterministe et statique au profit d'une perspective cognitive où l'attention serait tournée vers la « fabrication de sens » (sensemaking).

Cette perspective traditionnelle associée au modèle orthodoxe de production de connaissances est certainement légitime, mais elle a également fait l'objet de nombreuses critiques au cours des dernières années, notamment parce qu'elle suppose la stabilité de l'objet et l'extériorité du sujet, deux caractéristiques jugées insoutenables en recherche sociale (Audet, Landry et Déry, 1986). Dans cette perspective où, notent les mêmes auteurs, les connaissances sont considérées comme définitives et cumulatives, l'accent est mis sur la recherche d'une vérité cachée plutôt que sur une quête de sens. Compte tenu des limites de la perspective objectiviste, on comprend mal qu'elle soit surreprésentée dans le domaine de la PME et de l'entrepreneuriat. Chaque approche a ses limites, bien sûr; mais le point de vue subjectiviste constitue une alternative qui ne devrait pas être rejetée (voir, entre autres, Cossette, 1994a). Suivant cette orientation, on reconnait d'entrée de jeu que la structure cognitive du sujet le guide dans son appréhension du réel, qu'il est actif dans sa relation avec l'environnement et qu'il ne fait pas que capter passivement ou même percevoir un monde extérieur qui s'imposerait à lui. L'attention est dirigée sur la façon dont il construit subjectivement la réalité, c'est-à-dire sur la manière dont il découpe le réel et sur les liens qu'il établit entre les différents éléments qui résultent de ce découpage. On cherche alors à comprendre des réalités considérées comme fondamentalement uniques et en perpétuelle transformation, en faisant appel aux représentations ou schèmes des acteurs concernés. L'objectif n'est plus de généraliser des faits reposant sur la mise au jour de supposées lois de la nature mais de susciter des prises de conscience permettant de mieux comprendre des situations particulières se produisant dans d'autres contextes ou organisations (Morgan, 1985). Méthodologiquement, une telle orientation implique l'adoption d'une approche idiographique. La stratégie de recherche doit permettre aux gens concernés de livrer, voire de construire, leur propre conception des choses, ce qui requiert habituellement l'emploi de techniques comme l'entrevue en profondeur, 
l'observation participante et l'analyse de contenu. Suivant cette perspective subjectiviste, une organisation, petite ou grande, ne peut être comprise sans faire appel à ce que pensent les acteurs concernés, et à la façon particulière dont ils le pensent. L'adoption de cette perspective par un plus grand nombre de chercheurs pourrait constituer l'un des facteurs les plus importants dans le développement des connaissances dans le champ de l'entrepreneuriat et de la PME.

\section{ANNEXE}

\section{Produits cognitifs $(n=119)$}

Banks et al., 1987, JSBM, 25(1)

Specht, 1987, AJSB (ETP), 11(4)

Nelson, 1987, JSBM, 25(3)

Jankowicz et Hisrich, 1987, JSBM, 25(3)

Schlesinger et al., 1987, JSBM, 25(4)

Schwartz, 1987, JEBO, 8(4)

Dart, 1988, AJSB (ETP), 12(3)

Keats et Bracker, 1988, AJSB (ETP), 12 (4)

Morganosky, 1988, JSBM, 26(1)

Corman et al., 1988, JSBM, 26 (1)

Pelham et Clayson, 1988, JSBM, 26(1)

Cooper et al., 1988, JBV, 3(2)

Hills, 1988, JBV, 3(2)

Buttner et Rosen, 1988, JBV, 3(3)

Greenberger et Sexton, 1988, JSBM, 26(3)

Raymond, 1988, JSBM, 26(3)

Smeltzer et al., 1988, JSBM, 26(3)

Katz et Gartner, 1988, AMR, 13(3)

Bird, 1988, AMR, 13(3)

Perry, 1988, AME

Scott et Twomey, 1988, JSBM

Kathawala, 1988, JORS, 39(11)

Bird, 1988, ETP, 13(2)

Dubini, 1989, JBV, 4(1)

Kathawala et al., 1989, JSBM, 27(1)

Smeltzer et Fann, 1989, JSBM, 27(2)

De Noble et al., 1989, JSBM, 27(2)

Sharkey et al., 1989, MIR, 29(2)

Davidsson, 1989, JBV, 4(3)

Nelson, 1989, ETP, 13(4)

Fann et Smeltzer, 1989, ETP, 13(4)

Dilts et Prough, 1989, JSBM, 27(3)

Wagner et Fulford, 1989, JSBM, 27(3)

Snyder et al., 1989, JSBM, 27(3)

Fann et Smeltzer, 1989, JBC, 26(4)

Aram, 1989, JBV, 4(5)

Christman, 1989, JBV, 4(6)

Evans et al., 1990, JSBM, 28(1)

Gartner, 1990, JBV, 5(1)

Hisrich et Jankowicz, 1990, JBV, 5(1)
Smilor et al., 1990, JBV, 5(1)

Ray et Turpin, 1990, JBV, 5(2)

Alpander et al., 1990, JSBM, 28(2)

Scherer et al., 1990, JSBM, 28(2)

Clouse, 1990, JSBM, 28(2)

Ohe et al., 1990, JBV, 5(3)

Lee-Gosselin et Grizé, 1990, JBE, 9(4,5)

Krackhardt, 1990, ASQ, 35(2)

Tang, 1990, IA, 12(3)

Miller, 1990, JBV, 5(4)

Peterson et Dant, 1990, JSBM, 28(3)

Lee et Mulford, 1990, JSBM, 28(3)

Keasey et Short, 1990, ABR, 20(80)

Gales et Blackburn, 1990, ETPM, 15(1)

Galbraith et al., 1990, JSBM 28(4)

Lynn et Reinsch, 1990, JSBM, 28(4)

Dean et Giglierano, 1990, JBV, 5(6)

Gul, 1991, ABR, 22(85)

Withane, 1991, JSBM, 29(1)

Larson et al., 1991, JSBM, 29(2)

Enz et al., 1990, ETP, 15(1)

Mayo, 1991, JSBM, 29(2)

Ali et Swiercz, 1991, JSBM, 29(2)

Clouse, 1991, T, 11(4)

Arlow et Ackelsberg, 1991, ABER, 22(2)

Fagenson et Marcus, 1991, ETP, 15(4)

Smeltzer et al., 1991, JSBM, 29(3)

Brenner et al., 1991, JSBM, 29(3)

Norton, 1991, JBV, 6(4)

Weinrauch et al., 1991, IMM, 20(3)

Caneday et Zeiger, 1991, JTR, 30(2)

Williams et al., 1991, JABR, 7(4)

Weinrauch et al., 1991, JSBM, 29(4)

Shane et al., 1991, JBV, 6(6)

Haines et al., 1991, JBF, 15(6)

Buttner et Rosen, 1992, JSBM, 30(1)

Silverman et Castaldi, 1992, JSBM, 30(2)

Kolvereid, 1992, JBV, 7(3)

Chandler et Janson, 1992, JBV, 7(3)

Neeley, 1992, JSBM, 30(3) 
Coker et Hayes, 1992, JSBM, 30(3)

Serwinek, 1992, JBE, 11(7)

Chawla et al., 1992, JABR, 8(4)

Campbell, 1992, ETP, 17(1)

Guptu et Sapienza, 1992, JBV, 7(5)

McGrath et MacMillan, 1992, JBV, 7(5)

Taylor et Banks, 1992, JSBM, 30(4)

Weinstein et al., 1992, JSBM, 30(4)

Smith et Hoy, 1992, JSBM, 30(4)

Manimala, 1992, JBV, 7(6)

Lasher et Grashof, 1993, JSBM, 31(1)

Hall et Hofer, 1993, JBV, 8(1)

Chua et Woodward, 1993, JBV, 8(1)

Rosenstein et al., 1993, JBV, 8(2)

Hood et Young, 1993, JBV, 8(2)

Cragg et King, 1993, MISQ, 17(1)

Terpstra et Olson, 1993, ETP, 17(3)

Arbuthnot et al., 1993, JSBM, 31(2)

Lee et Ackelsberg, 1993, JSBM, 31(2)

Minkler, 1993, JEBO, 21(1)
Baker et Cunningham 1993, JBFA, 20(4)

Bird et al., 1993, ETP, 17(4)

Fay et Williams, 1993, JBV, 8(4)

Humphreys et al., 1993, JSBM, 31(3)

Shama, 1993, JSBM, 31(3)

Krueger, 1993, ETP, 18(1)

Gaskill et al., 1993, JSBM, 31(4)

Kolvereid et al., 1993, JSBM, 31(4)

Reeves et Hoy, 1993, JSBM, 31(4)

Jones et Kohers, 1993, MF, 19(8)

Harman et al., 1994, JSBM, 32(1)

Carter et Van Auken, 1994, JSBM 32(1)

Birley et Westhead, 1994, JBV, 9(1)

Dodge et al., 1994, SMJ, 15(2)

Naffziger et al., ETP, 18(3)

Venkataraman et Low, 1994, JBV, 9(3)

Boyd et Vozikis, 1994, ETP, 18(4)

Smith, 1994, JEBO, 24(2)

Covin, 1994, JSBM, 32(3)

\section{Processus cognitifs $(n=11)$}

Johnson et Kuehn, 1987, JSBM, 25(3)

Smith et al., 1988, JBV, 3(3)

Feldman, 1988, OD, 17(1)

Sandberg et al., 1988, ETP, 13(2)

Alpander et al., 1990, JSBM, 28(2)

Bouwen et Steyaert, 1990, JMS, 27(6)
Shaver et Scott, 1991, ETP, 16(2)

Bird, 1992, ETP, 17(1)

Katz, 1992, ETP, 17(1)

Learned, 1992, ETP, 17(1)

Gifford, 1992, JEBO, 19(3)

\section{Prédispositions cognitives $(n=17)$}

Ulrich et Cole, 1987, JSBM, 25(4)

Longenecker et al., 1988, OD, 16(3)

Lafuente et Salas, 1989, SMJ, 10(1)

Rice et Lindecamp, 1989, JOP, 62(2)

Ginn et Sexton, 1990, JBV, 5(5)

Enz et al., 1990, ETP, 15(1)

Robinson et al., 1991, JSBM, 29(2)

Robinson et al., 1991, ETP, 15(4)

Bonnett et Furnham, 1991, JEP, 12(3)
Goldsmith et Kerr, 1991, T, 11(6)

Olson et Currie, 1992, JSBM, 30(1)

McGrath et al., 1992, JBV, 7(2)

Starr et Fondas, 1992, ETP, 17(1)

Buttner et Gryskiewicz, 1993, JSBM, 31(1)

Fagenson, 1993, JBV, 8(5)

Ramachandran et Ramnarayan, 1993, JBV, 8(6)

Huefner et Hunt, 1994, ETP, 18(3)

\section{Procédés cognitifs $(n=11)$}

\begin{tabular}{|ll|}
\hline Fairfield-Sonn, 1987, JSBM, 25(1) & Chen, 1989, JSBM, 27(3) \\
Gatewood et Feild, 1987, JSBM, 25(4) & Armacost et al., 1990, ABER, 21(1) \\
Gilmore et Kazanjian, 1989, JBV, 4(1) & Borch et Hartvigsen, 1991, DSS, 7(2) \\
Greer, 1989, JSBM, 27(1) & Martin et al., 1991, JSBM, 29(4) \\
Sullivan et Shively, 1989, JSBM, 27(1) & Cossette et Audet, 1992, JMS, 29(3) \\
& Cook et Barry, 1993, JSBM, 31(1) \\
& \\
\hline
\end{tabular}




\section{Bibliographie}

ANDLER, D. (dir.) (1992), Introduction aux sciences cognitives, Paris, Gallimard, collection «Folio/essais ».

AUDET, M. et R. DÉRY (1996), «La science réfléchie. Quelques empreintes de l'épistémologie des sciences de l'administration ", Anthropologie et Sociétés, vol. 20, $\mathrm{n}^{\circ} 1$, p. 103-123.

AUDET, M., M. LANDRY et R. DÉRY (1986), « Science et résolution de problème : liens, difficultés et voies de dépassement dans le champ des sciences de l'administration », Philosophy of the Social Sciences/Philosophie des sciences sociales, vol. 16, p. 409-440.

BROCKHAUS, R.H. SR. (1987), «Entrepreneurial research : are we playing the correct game? », American Journal of Small Business, vol. 11, $\mathrm{n}^{\circ} 3$, p. 43-49.

BULL, I. et G.E. WILLARD (1993), « Towards a theory of entrepreneurship », Journal of Business Venturing, vol. 8, $\mathrm{n}^{\circ}$ 3, p. 183-195.

BuSENITZ, L.W. et C.M. LAU (1996), «A cross-cultural cognitive model of new venture creation », Entrepreneurship Theory and Practice, vol. 20, n 4, p. 25-39.

BYGRAVE, W.D. (1989), « The entrepreneurship paradigm (I) : a philosophical look at its research methodologies », Entrepreneurship Theory and Practice, vol. 14, $\mathrm{n}^{\circ} 1$, p. 7-26.

BYGRAVE, W.D. (1993), «Theory building in the entrepreneurship paradigm », Journal of Business Venturing, vol. 8, no 3, p. 255-280.

BygraVE, W.D. et C.W. HOFER (1991), «Theorizing about entrepreneurship», Entrepreneurship Theory and Practice, vol. 16, $\mathrm{n}^{\circ}$ 2, p. 13-22.

CARRIER, C. (1996), «Intrapreneurship in small businesses : an exploratory study », Entrepreneurship Theory and Practice, vol. 21, $\mathrm{n}^{\circ} 1$, p. 5-20.

ChURCHILl, N.C. et V.L. LEWIS (1986), «Entrepreneurship research : directions and methods », dans D. L. Sexton et R. W. Smilor (dir.), The art and science of entrepreneurship, Cambridge, Mass., Ballinger.

COSSETTE, P. (1994a), «Les cartes cognitives au service de l'étude des organisations », dans P. Cossette (dir.), Cartes cognitives et organisations, p. 3-12, Québec, Les Presses de l’Université Laval; Paris, Éditions Eska, collection «Sciences de l'administration ».

CosSETTE, P. (1994b), «Structures cognitives et organisations », dans C. Louche (dir.), Individu et organisations, p. 154-177, Lausanne, Delachaux et Niestlé, collection «Textes de base en sciences sociales ».

COSSETTE, P. (1995), « La cognition comme objet d'étude dans la littérature scientique sur la PME et l'entrepreneuriat », Actes du II Congrès international francophone de la PME (CIFPME), Paris, p. 487-524.

CosseTte, P. (1997), «Les travaux publiés dans la Revue internationale PME depuis sa fondation : caractéristiques et tendances », Revue internationale PME, vol. 10, $\mathrm{n}^{\circ} 2$, p. 109-128. 
D'AMboise, G. et M. Muldowney (1988), « Management theory for small business : attempts and requirements », Academy of Management Review, vol. 13, $\mathrm{n}^{\circ} 2$, p. 226-240.

DANDRIDGE, T. C. (1979), « Children are not « little grown-ups» : small business needs its own organizational theory », Journal of Small Business Management, vol. 17, $\mathrm{n}^{\circ}$ 2, p. 53-57.

DÉRY, R. (1989), «La structuration discursive de la problématique de la décision dans la revue Administrative Science Quarterly: une contribution à l'épistémologie des sciences de l'organisation », Thèse de doctorat non publiée, Université Laval, Québec.

EVERED, R. et M.R. LOUIS (1981), «Alternative perspectives in the organizational sciences : "inquiry from the inside" and "inquiry from the outside" ", Academy of Management Review, vol. 6, p. 385-395.

HeRron, L., H.J. SAPIENZA et D. SMITH-COOK (1991), «Entrepreneurship theory from an interdisciplinary perspective : volume I », Entrepreneurship Theory and Practice, vol. $16, \mathrm{n}^{\circ} 2$, p. $7-12$.

Herron, L., H.J. SAPIEnZa et D. SMith-CoOK (1992), «Entrepreneurship theory from an interdisciplinary perspective : volume II », Entrepreneurship Theory and Practice, vol. $16, \mathrm{n}^{\circ} 3$, p. 5-11.

HISRICH, R.D. (1988), «Entrepreneurship : past, present, and future », Journal of Small Business Management, vol. 26, $\mathrm{n}^{\circ}$ 4, p. 1-4.

Hofer, C.W. et W.D. BygRAVE (1992), «Researching entrepreneurship», Entrepreneurship Theory and Practice, vol. 16, $\mathrm{n}^{\circ} 3$, p. 91-100.

HuFf, A. S. (dir.) (1990), Mapping Strategic Thought, Chichester, John Wiley and Sons.

IRELAND, R.D. et P.M. VAN AUKEN (1987), «Entrepreneurship and small business research : an historical typology and directions for future research », American Journal of Small Business, vol. 11, $\mathrm{n}^{\circ}$ 4, p. 9-20.

JELINEK, M. et J.A. LITTERER (1995), « Toward entrepreneurial organizations : meeting ambiguity with engagement », Entrepreneurship Theory and Practice, vol. 19, n ${ }^{\circ} 3$, p. 137-168.

LOW, M.B. et I.C. MACMILLAN (1988), «Entrepreneurship : past research and future challenges », Journal of Management, vol. 14, $\mathrm{n}^{\circ}$ 2, p. 139-161.

MACMILLAN, I.C. (1993), « The emerging forum for entrepreneurship scholars », Journal of Business Venturing, vol. 8, $\mathrm{n}^{\circ} 5$, p. 377-381.

MACMILLAN, I. C. et J.A. KATZ (1992), «Idiosyncratic milieus of entrepreneurial research : the need for comprehensive theories ", Journal of Business Venturing, vol. 7, $\mathrm{n}^{\circ} 1$, p. $1-8$.

MeindL, J.R., C. Stubbart et J.F. PoraC (1994), «Cognition within and between organizations : five key questions », Organization Science, vol. 5, $\mathrm{n}^{\circ} 3$, p. 289-293.

MORGAN, G. (1985), «Qualitative and action based research», Actes du colloque "Perspective de recherche pour le praticien », Rouyn, Université du Québec en AbitibiTémiscamingue, p. 81-109. 
ROMANO, C.A. (1989), « Research strategies for small business : a case study approach », International Small Business Journal, vol. 7, $\mathrm{n}^{\circ} 4$, p. 35-43.

SANDBERG, W.R. et E.J. GATEWOOD (1991), «A profile of entrepreneurship research centers : orientations, interests, activities, and resources », Entrepreneurship Theory and Practice, vol. 15, $\mathrm{n}^{\circ}$ 3, p. 11-24.

SCHNEIDER, S.C. et R. ANGELMAR (1993), « Cognition in organizational analysis : who's minding the store ?», Organization Studies, vol. 14, $\mathrm{n}^{\circ} 3$, p. 347-374.

SEXTON, D.L. (1987), «Advancing small business research : utilizing research from other areas », American Journal of Small Business, vol. 11, n 3, p. 25-30.

SIMS, H.P. JR. et D.A. GIOIA (dir.) (1986), The Thinking Organization: Dynamics of Organizational Social Cognition, San Francisco, Jossey-Bass.

SMIRCICH, L. et C.I. STUBBART (1985), « Strategic management in an enacted world», Academy of Management Review, vol. 10, $\mathrm{n}^{\circ}$ 4, p. 724-736.

STEVENSON, H. et S. HARMELING (1990), «Entrepreneurial management's need for a more "chaotic" theory ", Journal of Business Venturing, vol. 5, $\mathrm{n}^{\circ}$ 1, p. 1-14.

VESPER, K.H. (1987), «Entrepreneurial academics - how can we tell when the field is getting somewhere », Journal of Small Business Management, vol. 25, $\mathrm{n}^{\circ}$ 2, p. 1-7.

WEICK, K.E. (1979), The Social Psychology of Organizing, $2^{\mathrm{e}}$ éd., Reading, Mass., AddisonWesley.

WELSH, J.A. et J.F. WHITE (1981), « A small business is not a little big business », Harvard Business Review, vol. 59, n ${ }^{\circ}$ 4, p. 18-32.

WORTMAN, M.S. JR. (1986), «A unified framework, research typologies, and research prospectuses for the interface between entrepreneurship and small business », dans D.L. Sexton et R.W. Smilor (dir.), The art and science of entrepreneurship, Cambridge, Mass., Ballinger, p. 273-331.

WORTMAN, M.S. JR. (1987), «Entrepreneurship : an integrating typology and evaluation of the empirical research in the field », Journal of Management, vol. 13, $\mathrm{n}^{\circ} 2$, p. 259-279. 